CLINICAL STUDY

\title{
Lack of influence of somatic mutations on steroid gradients during adrenal vein sampling in aldosterone-producing adenoma patients
}

Andrea Oßwald, Evelyn Fischer, Christoph Degenhart ${ }^{1}$, Marcus Quinkler ${ }^{2}$, Martin Bidlingmaier, Anna Pallauf, Katharina Lang ${ }^{3}$, Thomas Mussack ${ }^{4}$, Klaus Hallfeldt ${ }^{4}$, Felix Beuschlein and Martin Reincke

Medizinische Klinik und Poliklinik IV, Ludwig-Maximilians-Universität München, Klinikum der Universität München, Ziemssenstr. 1, D-80336 Munich, Germany, ${ }^{1}$ Institut für Klinische Radiologie, Ludwig-Maximilians-Universität München, Munich, Germany, ${ }^{2}$ Klinische Endokrinologie Charité Campus Mitte, Universitätsmedizin Berlin, Berlin, Germany, ${ }^{3}$ Endocrine and Diabetes Unit, Department of Internal Medicine I, University of Würzburg, Würzburg, Germany and ${ }^{4}$ Chirurgische Klinik und Poliklinik - Innenstadt, Klinikum der Ludwig-Maximilians-Universität München, Munich, Germany

(Correspondence should be addressed to M Reincke; Email: martin.reincke@med.uni-muenchen.de)

\begin{abstract}
Objective: Adrenal vein sampling (AVS) is a technically demanding procedure required for the identification of suitable candidates for unilateral adrenalectomy in primary aldosteronism. Recently, somatic KCNJ5 $\mathrm{K}^{+}$-channel mutations in aldosterone-producing adenoma (APA) patients have been shown to influence steroid gradients during AVS. These and other recently identified genetic modifiers (ATP1A1 and ATP2B3) might affect the final diagnosis and treatment of the affected patients.

Design: Fifty-nine patients with APAs who had undergone successful AVS (adrenal vein cortisol:peripheral cortisol ratio $\geq 2$ ) and had undergone a mutation analysis of their tumor tissue were studied. The mutation status of the APAs was as follows: 19 KCNJ5 mutations, eight ATPase mutations (five ATP1A1 and three ATP2B3), and 32 patients with none of these mutations.

Methods: The lateralization index (ratio of aldosterone:cortisol on the side of the adenoma to aldosterone to cortisol on the contralateral side) and the contralateral suppression index (ratio of aldosterone:cortisol on the contralateral side to aldosterone to cortisol in the periphery) were calculated for the KCNJ5-mutated, ATPase-mutated, and the KCNJ5/ATPase mutation-negative APA patients.

Results: The lateralization indices of the ATPase mutation carriers had a median of 19.9 compared with a median of 16.0 in the KCNJ5 mutation carriers and that of 20.5 in the KCNJ5/ATPase mutation-negative patients. The contralateral suppression indices of the ATPase-mutated patients had a median of 0.1 compared with a median of 0.4 in the KCNJ5 mutation carriers and that of 0.2 in the KCNJ5/ATPase mutation-negative patients. The differences between the genetic groups were not statistically significant.

Conclusions: We did not find evidence for a clinically important impact of mutation status on steroid gradients during AVS.
\end{abstract}

European Journal of Endocrinology 169 657-663

\section{Introduction}

Primary aldosteronism (PA) is the most common cause of secondary arterial hypertension. It has been reported to occur in $\sim 7 \%$ of hypertensive patients in populationbased studies and in up to $20 \%$ of those with resistant hypertension in specialized centers $(1,2)$. Cardiovascular and renal morbidities are increased in patients with PA compared with patients with essential hypertension $(3,4,5)$. Therefore, early diagnosis and specific therapy are of crucial importance (6). The two main causes of PA are aldosterone-producing adenomas (APAs) and bilateral idiopathic adrenal hyperplasia. While APAs can effectively be cured with unilateral adrenalectomy, bilateral idiopathic adrenal hyperplasia is treated with lifelong therapy with mineralocorticoid receptor antagonists. Subtype differentiation between unilateral adrenal disease and bilateral adrenal disease necessitates adrenal vein sampling (AVS) (7).

Recently, somatic mutations in APA patients have become a focus of research. In 2011, Choi et al. (8) discovered mutations in the KCNJ5 gene, coding for the potassium channel KIR 3.4 , in $36 \%$ of the sporadic APA cases. The detected mutations were localized near the selectivity filter of the channel. Thus, KCNJ5-mutated potassium channels lose their ion selectivity and permit sodium influx, leading to the depolarization of the cell (9). This leads to the opening of voltage-dependent calcium channels and influx of calcium. The enhanced calcium concentration induces aldosterone production 
via the calcium signaling cascade. In many consecutive studies including our own series, affected patients were demonstrated to be predominantly females (10). We have recently discovered somatic mutations in the $\mathrm{Na}^{+}$, $\mathrm{K}^{+}$-ATPase (ATP1A1) and $\mathrm{Ca}^{2+}$-ATPase (ATP2B3) genes in 5.2 and $1.6 \%$ of the APA patients respectively (11). These mutations were localized in the ion-binding pocket. Functional studies of ATP1A1 mutations have demonstrated the loss of function of the pump and depolarization of the cells. Patients affected by these mutations are predominantly males and have a more severe endocrine and cardiovascular phenotype.

AVS is a technically demanding procedure that is required for the identification of suitable candidates for unilateral adrenalectomy in PA. A recent study has shown that steroid gradients during AVS are influenced by the KCNJ 5 mutation status of the APA (12). However, the impact of ATPase mutations has not been studied yet. This might have an impact on final diagnosis and treatment.

\section{Subjects and methods}

\section{Patient cohort}

Patients with APAs were recruited consecutively in three different German centers (Munich, $n=50$; Berlin, $n=8$; and Würzburg, $n=1$ ) of the German Conn's Registry - Else Kröner-Fresenius Hyperaldosteronismus Registry (www.conn-register.de). Of the 59 patients recruited, 46 were part of the study carried out by Beuschlein et al. (11). Case detection and subtype identification of PA were carried out according to institutional guidelines and in accordance with the Endocrine Society Guidelines $(7,13)$. The final diagnosis of APAs was based on the following criteria: biochemical diagnosis of hyperaldosteronism, lateralization of aldosterone production during AVS, histological confirmation of adrenocortical adenomas and normalization of hypokalemia, hypertension, and aldosteroneto-renin ratio after adrenalectomy $(14,15)$. The Ethics Committee of the participating centers approved the protocol of the German Conn's Registry, and all patients provided written informed consent for genetic and clinical investigations.

\section{Adrenal vein sampling}

Before AVS, the use of interfering medications such as mineralocorticoid receptor antagonists, diuretics, and $\beta$-blockers was stopped. Instead, verapamil (maximum dose of $240 \mathrm{mg}$ twice daily) and doxazosin (maximum dose of $16 \mathrm{mg}$ daily) were used and hypokalemia was corrected. AVS was carried out between 0800 and $1200 \mathrm{~h}$ by experienced radiologists. Blood samples were sequentially collected from both adrenal veins without adrenocorticotrophic hormone (ACTH) stimulation.
Hydrophilic 4 French catheters with different configurations were used, depending on the anatomy of the adrenal veins. The samples were collected by gravity or gentle suction. Corresponding peripheral samples were collected at the time of AVS of each adrenal vein $(n=48)$ or only once during AVS $(n=11)$. The mean selectivity index of the two groups was similar.

A selectivity index (adrenal vein cortisol to peripheral cortisol) of at least 2 on both sides and a lateralization index (ratio of aldosterone:cortisol on the side of the adenoma to aldosterone to cortisol on the contralateral side) of 4 or above were set for the diagnosis of unilateral aldosterone excess (16). The contralateral suppression index was calculated as the ratio of the cortisolcorrected aldosterone ratio (AC) of the nondominant adrenal gland to the peripheral $\mathrm{AC}$ $\left(A C_{\text {nondominant adrenal }} / A C_{\text {periphery }}\right)$. The decision for adrenalectomy was not based on the contralateral suppression of aldosterone secretion.

\section{Biochemical measurements}

Plasma aldosterone concentration was measured using Coat-a-Count RIA (Biermann DPC, Bad Nauheim, Germany). Active renin concentration was measured using the Diasorin assay (Liaison, Saluggia, Italy) in Munich and Würzburg and using the Cisbio assay (Berlin, Germany) in Berlin. In this study, the respective within-assay and between-assay coefficients of variation were below 9 and 12\% for aldosterone and below 5.6 and $12.2 \%$ for renin respectively. All other biochemical variables were assayed using plasma or serum in our central laboratory using standard methods. Serum potassium concentration was measured using flame photometry (ISE Indirect, Cobas Integra, the Roche platform; Roche).

\section{KCNJ5, ATP1A1, and ATP2B3 sequencing}

DNA was extracted from APA tissue using the RNeasy DNA extraction kit (Qiagen) and amplified using intronspanning primers as described previously $(8,11)$. Bi-directional Sanger sequencing was carried out using the ABI 3730xl Analyzer.

\section{Statistical analysis}

Data were extracted from the German Conn's Registry Else Kröner-Fresenius Hyperaldosteronismus Registry. If not stated otherwise, group results are reported as medians and interquartile ranges (IQRs). Data of the groups were compared using the Kruskal-Wallis test followed by a two-sided test for pairwise comparison of two groups. Power calculation of the study was based on the data of the study carried out by Seccia et al. (12) and on a conservative assumption of mean lateralization indices of 30 and 15 (s.D. \pm 15 ) in mutated vs nonmutated APA patients. This estimation required 







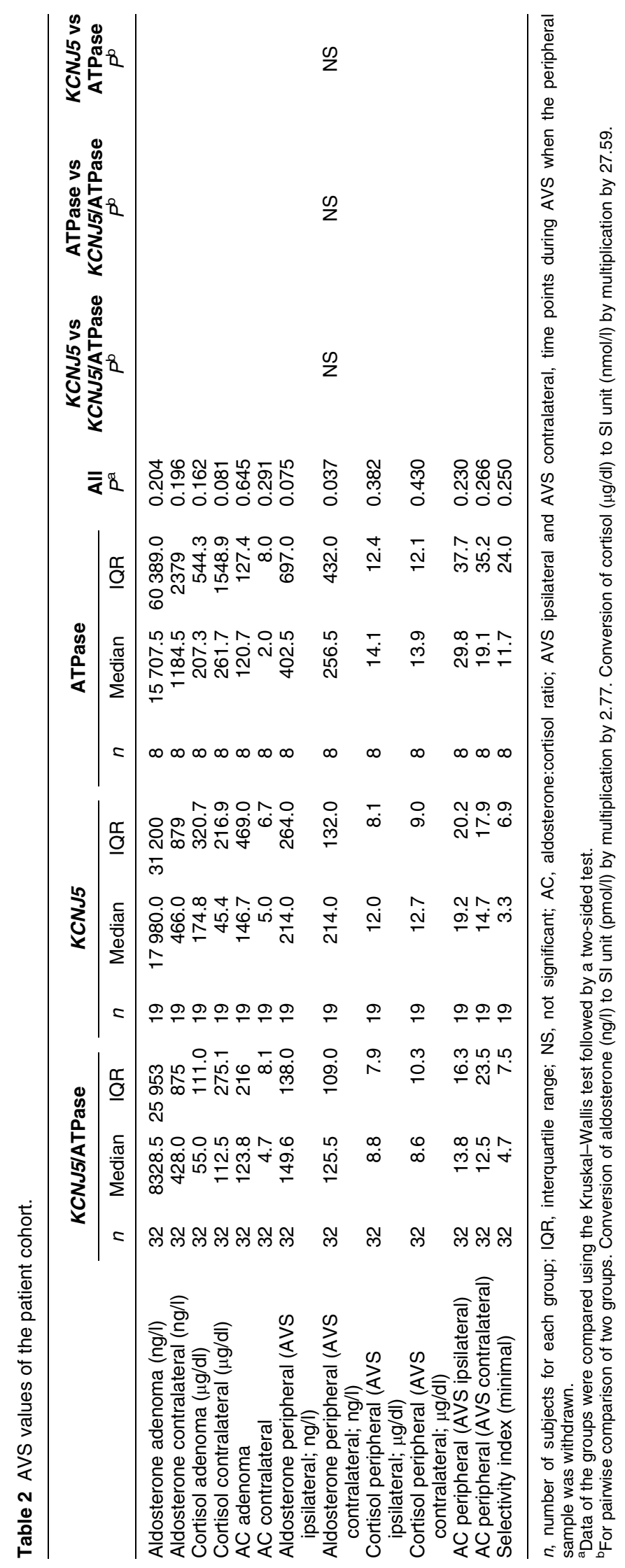


17 patients in each group to detect a significant difference $(P<0.05)$ with a power of $80 \%$. Statistical analysis was carried out using the standard statistical software (SPSS 21).

\section{Results}

To analyze the potential influence of somatic mutations on steroid gradients during AVS, 59 consecutive patients with APAs in three German centers were prospectively studied. Among the patients, 37 were men and 22 were women. All the subjects were diagnosed according to the German Conn's Registry standard. Following genetic analysis of the adenoma tissue, it was found that 19 patients had KCNJ5 mutations (11 G151R and eight L168R) and eight had ATPase mutations (five ATP1A1 and three ATP2B3), while 32 APA patients did not harbor any of these mutations.

The baseline and follow-up characteristics of the cohort are summarized in Table 1. As has been reported previously, patients with KCNJ5 mutations were predominantly females and younger at the time of surgery, whereas patients with ATPase mutations displayed a male predominance and were older.

AVS was carried out without ACTH stimulation. The AVS values are summarized in Table 2, and individual values are given in Supplementary Table 1, see section on supplementary data given at the end of this article.

To analyze the lateralization of aldosterone production during AVS, the lateralization index was calculated. The lateralization index of the nonmutated and KCNJ5-mutated patients had a median of 20.5 (IQR 30.3) and 16.0 (IQR 41.9) respectively (Fig. 1A). The ATPase mutation carriers had a median lateralization index of 19.9 (IQR 122.3). These differences between the genetic groups were not significant $(P=0.959)$.

Contralateral suppression was most distinct in the ATPase-mutated patients with a median of 0.1 (IQR 0.3 ) compared with a median of 0.4 (IQR 0.5 ) in the KCNJ5-mutated patients and that of 0.2 (IQR 0.6) in the nonmutated patients (Fig. 1B), but these differences were not significant $(P=0.060)$.

\section{Discussion}

AVS is the recommended procedure for the identification of patients with APAs that can be cured with unilateral adrenalectomy. However, it is unclear whether the mutation status of APAs affects steroid gradients during AVS. Seccia et al. (12) have reported on the impact of KCNJ5 mutations on aldosterone gradients during AVS and have found a more profound lateralization index in their cohort. These data have not been confirmed in independent cohorts, and the newly
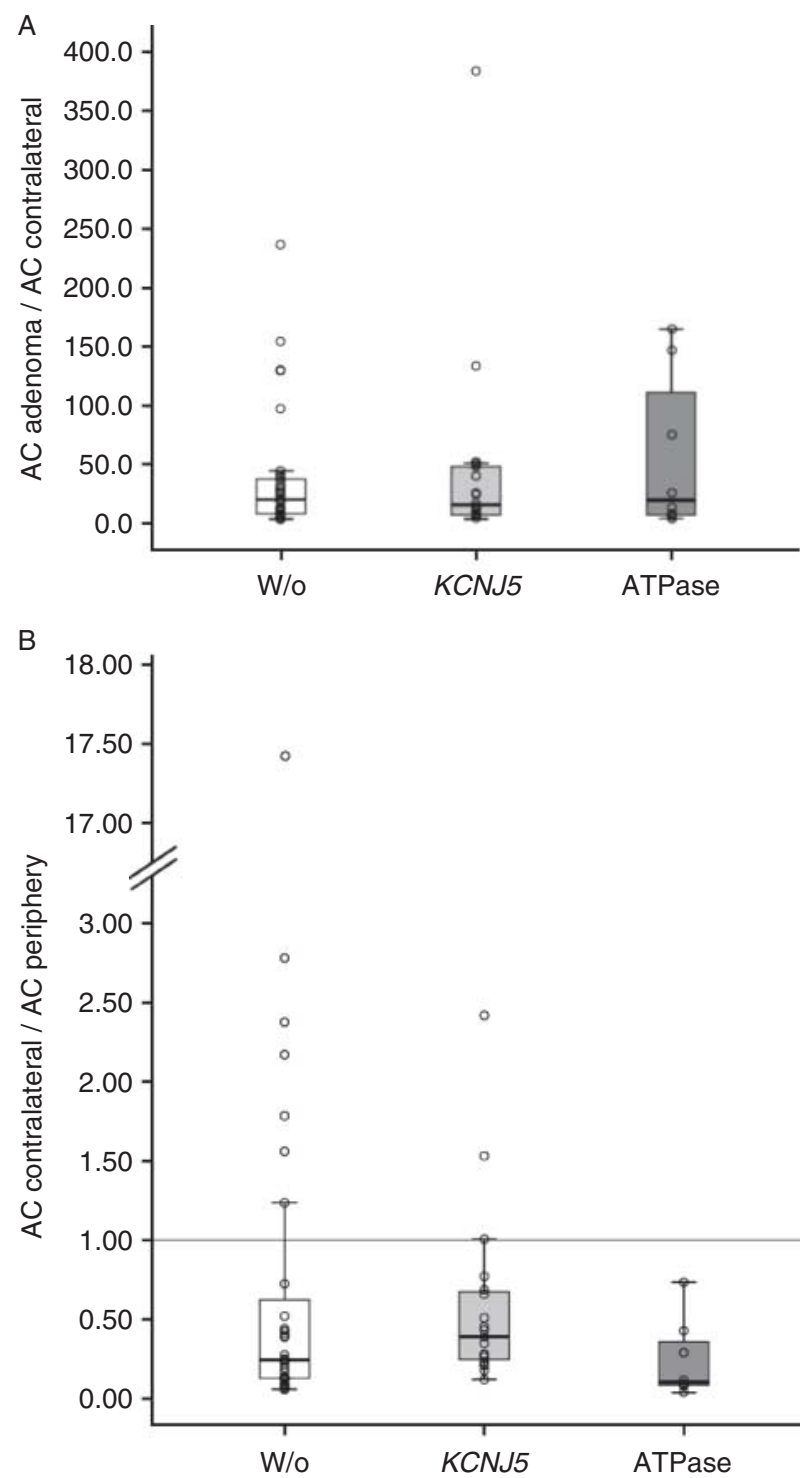

Figure $1(A)$ Lateralization index. The box and whisker plot (median and 25-75th percentiles) shows the ratio of cortisol-normalized aldosterone on the APA side:cortisol-normalized aldosterone on the contralateral side for KCNJ5/ATPase-nonmutated (w/o) patients and for the KCNJ5- and ATPase-mutated patients. (B) Suppression index. The box and whisker plot (median and 25-75th percentiles) shows the ratio of cortisol-normalized aldosterone on the contralateral side:cortisol-normalized aldosterone in the periphery for the KCNJ5/ATPase-nonmutated (w/o) patients and for the KCNJ5- and ATPase-mutated patients.

identified ATPase mutations have not been appreciated in this context yet.

Only patients who had undergone technically successful AVS (selectivity index $\geq 2.0$ ) exhibiting a lateralization of aldosterone production (lateralization index $\geq 4$.0) were included in this study as these were set as the criteria for unilateral adrenalectomy within the German Conn's Registry. The patient cohort 
displayed a composition similar to the ones reported previously (17). Among the subjects, $32 \%$ had KCNJ5 mutations and $14 \%$ had ATPase mutations. Gender distribution and age at diagnosis also resembled published data (10).

In this study, the lateralization indices of the nonmutated and ATPase-mutated patients were nearly equal and were higher than those of the KCNJ5 mutation carriers. This finding is in contrast to the findings of the study of Seccia et al., who demonstrated that KCNJ5-mutated patients have a significantly higher lateralization index than the KCNJ5 mutation-negative patients. This discrepancy might be explained by different inclusion criteria for their patient cohort based on a selectivity index of 2 and a lateralization index of 2, impeding the direct comparison of the two studies $(12,18)$. A lateralization index of 4 , as used in the present study, would have placed $68 \%$ of the KCNJ5 mutation-negative patients and $24 \%$ of the KCNJ5 mutation-positive cases of the Seccia study in the category of bilateral adrenal hyperplasia. These patients probably would not have been adrenalectomized in our clinical setting. Notably, the KCNJ5-mutated patients in the study carried out by Seccia et al. exhibited a more severe phenotype with higher aldosterone levels and lower potassium levels than our patients. One speculation might be that this cohort was diagnosed later and suffered longer from hyperaldosteronism than our patients. On the other hand, the Italian KCNJ5 mutation-negative patients seemed to exhibit a less severe phenotype with higher potassium levels and lower blood pressure in comparison with our nonmutated patients, despite the fact that within this group an unknown proportion of yet undiagnosed ATPase mutation carriers might have been included. In this context, it is not surprising if this group has high lateralization indices similar to the KCNJ5-mutated patients.

We found contralateral suppression to be most distinct in the ATPase-mutated patients. In general, a low contralateral suppression index indicates a more pronounced inhibition of aldosterone production from the contralateral adrenal gland. In our series, all the ATPase-mutated patients displayed full contralateral suppression $(100 \%)$, whereas only $84 \%$ of the KCNJ5mutated patients and $78 \%$ of the nonmutated patients had suppression indices below 1 . In the patient cohort of Seccia et al., 74\% of the KCNJ5-mutated APA patients exhibited contralateral suppression compared with 54\% of the KCNJ5 mutation-negative APA patients. These findings again support the hypothesis that the nonmutated patients in our cohort are different from the ones of the study of Seccia et al.

In summary, AVS is a clinically accurate method in the presence of APAs with or without KCNJ5 and ATPase mutations. We did not find convincing evidence for a clinically important impact of KCNJ5 and ATPase mutations on steroid gradients during AVS.

\section{Supplementary data}

This is linked to the online version of the paper at http://dx.doi.org/10. 1530/EJE-13-0551.

\section{Declaration of interest}

The authors declare that there is no conflict of interest that could be perceived as prejudicing the impartiality of the research reported.

\section{Funding}

This work was made possible by a grant from the Else KrönerFresenius-Stiftung in support of the German Conn's Registry - Else Kröner-Fresenius Hyperaldosteronism Registry (to M Reincke). Further funding was provided by the Deutsche Forschungsgemeinschaft to M Reincke and F Beuschlein (Re 752/17-1).

\section{Acknowledgements}

The authors thank the Munich PA team (Susanne Schmid, Friederike Konrad, and Gabriele Breu), without whose support this work would not have been feasible. They are indebted to Brigitte Mauracher for technical assistance.

\section{References}

1 Eide IK, Torjesen PA, Drolsum A, Babovic A \& Lilledahl NP. Low-renin status in therapy-resistant hypertension: a clue to efficient treatment. Journal of Hypertension 200422 2217-2226. (doi:10.1097/00004872-200411000-00026)

2 Hannemann A, Bidlingmaier M, Friedrich N, Manolopoulou J, Spyroglou A, Volzke H, Beuschlein F, Seissler J, Rettig R, Felix SB et al. Screening for primary aldosteronism in hypertensive subjects: results from two German epidemiological studies. European Journal of Endocrinology $2012 \quad \mathbf{1 6 7} 7-15 . \quad$ (doi:10.1530/ EJE-11-1013)

3 Born-Frontsberg E, Reincke M, Rump LC, Hahner S, Diederich S, Lorenz R, Allolio B, Seufert J, Schirpenbach C, Beuschlein F et al. Cardiovascular and cerebrovascular comorbidities of hypokalemic and normokalemic primary aldosteronism: results of the German Conn's Registry. Journal of Clinical Endocrinology and Metabolism 200994 1125-1130. (doi:10.1210/jc.2008-2116)

4 Catena C, Colussi G, Nadalini E, Chiuch A, Baroselli S, Lapenna R \& Sechi LA. Cardiovascular outcomes in patients with primary aldosteronism after treatment. Archives of Internal Medicine 2008 168 80-85. (doi:10.1001/archinternmed.2007.33)

5 Milliez P, Girerd X, Plouin PF, Blacher J, Safar ME \& Mourad JJ. Evidence for an increased rate of cardiovascular events in patients with primary aldosteronism. Journal of the American College of Cardiology $2005 \mathbf{4 5}$ 1243-1248. (doi:10.1016/j.jacc.2005. 01.015)

6 Catena C, Colussi G, Lapenna R, Nadalini E, Chiuch A, Gianfagna P \& Sechi LA. Long-term cardiac effects of adrenalectomy or mineralocorticoid antagonists in patients with primary aldosteronism. Hypertension $2007 \mathbf{5 0} 911-918$. (doi:10.1161/ HYPERTENSIONAHA.107.095448)

7 Funder JW, Carey RM, Fardella C, Gomez-Sanchez CE, Mantero F, Stowasser M, Young WF Jr \& Montori VM. Case detection, diagnosis, and treatment of patients with primary aldosteronism: an Endocrine Society clinical practice guideline. Journal of Clinical Endocrinology and Metabolism 200893 3266-3281. (doi:10.1210/jc.2008-0104)

8 Choi M, Scholl UI, Yue P, Bjorklund P, Zhao B, Nelson-Williams C, Ji W, Cho Y, Patel A, Men CJ et al. $\mathrm{K}^{+}$channel mutations in adrenal aldosterone-producing adenomas and hereditary hypertension. Science 2011331 768-772. (doi:10.1126/science.1198785) 
9 Mulatero P, Monticone S, Rainey WE, Veglio F \& Williams TA. Role of KCNJ5 in familial and sporadic primary aldosteronism. Nature Reviews. Endocrinology 20139 104-112. (doi:10.1038/nrendo. 2012.230)

10 Boulkroun S, Beuschlein F, Rossi GP, Golib-Dzib JF, Fischer E Amar L, Mulatero P, Samson-Couterie B, Hahner S, Quinkler M et al. Prevalence, clinical, and molecular correlates of $\mathrm{KCNJ} 5$ mutations in primary aldosteronism. Hypertension $2012 \mathbf{5 9}$ 592-598. (doi:10.1161/HYPERTENSIONAHA.111.186478)

11 Beuschlein F, Boulkroun S, Osswald A, Wieland T, Nielsen HN, Lichtenauer UD, Penton D, Schack VR, Amar L, Fischer E et al. Somatic mutations in ATP1A1 and ATP2B3 lead to aldosteroneproducing adenomas and secondary hypertension. Nature Genetics 201345 440-444. (doi:10.1038/ng.2550)

12 Seccia TM, Mantero F, Letizia C, Kuppusamy M, Caroccia B, Barisa M, Cicala MV, Miotto D \& Rossi GP. Somatic mutations in the KCNJ5 gene raise the lateralization index: implications for the diagnosis of primary aldosteronism by adrenal vein sampling. Journal of Clinical Endocrinology and Metabolism 2012 97 E2307-E2313. (doi:10.1210/jc.2012-2342)

13 Fischer E, Beuschlein F, Bidlingmaier M \& Reincke M. Commentary on the Endocrine Society practice guidelines: consequences of adjustment of antihypertensive medication in screening of primary aldosteronism. Reviews in Endocrine $\mathcal{E}$ Metabolic Disorders 201112 43-48. (doi:10.1007/s11154-011-9163-7)

14 Mulatero P, Bertello C, Verhovez A, Rossato D, Giraudo G, Mengozzi G, Limerutti G, Avenatti E, Tizzani D \& Veglio F. Differential diagnosis of primary aldosteronism subtypes. Current Hypertension Reports 200911 217-223. (doi:10.1007/s11906-009-0038-1)
15 Rossi GP, Bernini G, Caliumi C, Desideri G, Fabris B, Ferri C, Ganzaroli C, Giacchetti G, Letizia C, Maccario M et al. A prospective study of the prevalence of primary aldosteronism in 1,125 hypertensive patients. Journal of the American College of Cardiology 200648 2293-2300. (doi:10.1016/j.jacc.2006.07.059)

16 Betz MJ, Degenhart C, Fischer E, Pallauf A, Brand V, Linsenmaier U, Beuschlein F, Bidlingmaier M \& Reincke M. Adrenal vein sampling using rapid cortisol assays in primary aldosteronism is useful in centers with low success rates. European Journal of Endocrinology 2011165 301-306. (doi:10.1530/EJE11-0287)

17 Akerstrom T, Crona J, Delgado Verdugo A, Starker LF, Cupisti K, Willenberg HS, Knoefel WT, Saeger W, Feller A, Ip J et al. Comprehensive re-sequencing of adrenal aldosterone producing lesions reveal three somatic mutations near the KCNJ5 potassium channel selectivity filter. PLoS ONE 20127 e41926. (doi:10.1371/journal.pone.0041926)

18 Rossi GP, Barisa M, Allolio B, Auchus RJ, Amar L, Cohen D, Degenhart C, Deinum J, Fischer E, Gordon R et al. The Adrenal Vein Sampling International Study (AVIS) for identifying the major subtypes of primary aldosteronism. Journal of Clinical Endocrinology and Metabolism 201297 1606-1614. (doi:10.1210/jc.2011-2830)

Received 2 July 2013

Revised version received 8 August 2013

Accepted 12 August 2013 